\title{
AN INVERSE PROBLEM IN NUMBER THEORY AND GEOMETRIC GROUP THEORY
}

\author{
MELVYN B. NATHANSON
}

\begin{abstract}
This paper describes a new link between combinatorial number theory and geometry. The main result states that $A$ is a finite set of relatively prime positive integers if and only if $A=(K-K) \cap \mathbf{N}$, where $K$ is a compact set of real numbers such that for every $x \in \mathbf{R}$ there exists $y \in K$ with $x \equiv y$ $(\bmod 1)$. In one direction, given a finite set $A$ of relatively prime positive integers, the proof constructs an appropriate compact set $K$ such that $A=$ $(K-K) \cap \mathbf{N}$. In the other direction, a strong form of a fundamental result in geometric group theory is applied to prove that $(K-K) \cap \mathbf{N}$ is a finite set of relatively prime positive integers if $K$ satisfies the appropriate geometrical conditions. Some related results and open problems are also discussed.
\end{abstract}

\section{From COMPACT SETS TO INTEGERS}

The object of this note is to describe a new connection between number theory and geometry. Let $\mathbf{R}, \mathbf{Z}$, and $\mathbf{N}$ denote the real numbers, integers, and positive integers, respectively. For every $x \in \mathbf{R}$, let $[x] \in \mathbf{Z}$ and $(x) \in[0,1)$ denote the integer part and fractional part of $x$. Let $\mathbf{Z}^{n}$ denote the additive group of $n$ dimensional lattice points in the Euclidean space $\mathbf{R}^{n}$.

We recall the following definitions. The set $A$ of integers is relatively prime, denoted $\operatorname{gcd}(A)=1$, if $A$ is nonempty and the elements of $A$ have no common factor greater than 1. Equivalently, $A$ is relatively prime if $A$ generates the additive group $\mathbf{Z}$. The set $A$ of $n$-dimensional lattice points is relatively prime if the elements of $A$ generate the additive group $\mathbf{Z}^{n}$.

Let $H$ be a subgroup of a multiplicative group $G$, and let $x$ and $y$ be elements of $G$. We say that $x$ and $y$ are congruent modulo $H$, denoted $x \equiv y(\bmod H)$, if $x y^{-1} \in H$. If the group $G$ is additive, then $x \equiv y(\bmod H)$ if $x-y \in H$. For example, let $G=\mathbf{R}$ and $H=\mathbf{Z}$. The real numbers $x$ and $y$ are congruent modulo $\mathbf{Z}$, that is, $x \equiv y(\bmod \mathbf{Z})$ or, in more traditional notation, $x \equiv y(\bmod 1)$, if and only if they have the same fractional part.

In a multiplicative group $G$ with identity $e$, the difference set of a nonempty subset $K$ of $G$ is

$$
K K^{-1}=\left\{x y^{-1}: x, y \in K\right\}
$$

Date: November 12, 2018.

2000 Mathematics Subject Classification. Primary 11A05, 11B75, 11P21, 20 F65.

Key words and phrases. Relatively prime integers, combinatorial number theory, additive number theory, geometric group theory.

Supported in part by a grant from the PSC-CUNY Research Award Program. This paper was written while the author was a visiting fellow in the mathematics department at Princeton University. 
In an additive abelian group $G$, the difference set of a subset $K$ of $G$ is

$$
K-K=\{x-y: x, y \in K\} .
$$

Note that $e \in K K^{-1}$ and that the difference set is symmetric: $z \in K K^{-1}$ if and only if $z^{-1} \in K K^{-1}$ (resp. $z \in K-K$ if and only if $-z \in K-K$ ).

We are interested in sets of integers contained in difference sets of sets of real numbers. Our main theorem gives a geometric condition for a finite set of positive integers to be relatively prime. The geometry uses the concept of an $\mathcal{N}$-set, which is a compact subset $K$ of $\mathbf{R}^{n}$ such that for every $x \in \mathbf{R}^{n}$ there exists $y \in K$ with $x \equiv y\left(\bmod \mathbf{Z}^{n}\right)$.

Theorem 1. Let $A$ be a finite set of positive integers. The set $A$ is relatively prime if and only if there exists an $\mathcal{N}$-set $K$ in $\mathbf{R}$ such that $A=(K-K) \cap \mathbf{N}$.

In Section 2, we solve the inverse problem: Given a finite set of relatively prime integers, we construct an $\mathcal{N}$-set $K$ in $\mathbf{R}$ such that $A=(K-K) \cap \mathbf{N}$. In Section 3 we prove that if $K$ is an $\mathcal{N}$-set in $\mathbf{R}$, then $A=(K-K) \cap \mathbf{N}$ is a finite set of relatively prime positive integers. The proof uses the "fundamental observation of geometric group theory" (Theorem 5), which is reviewed in Section A.

Ideas from geometric group theory have been used recently to obtain new results in number theory (e.g. Nathanson [4, 5, 6]), and should continue to be useful. The book of de la Harpe, Topics in Geometric Group Theory [1, is an excellent survey of this subject. Theorem 5 was discovered and proved independently by Efremovič [2], Švarc [7, and Milnor [3].

\section{THE INVERSE PROBLEM}

In this section we prove that every finite set of relatively prime positive integers can be realized as the difference set of an $\mathcal{N}$-set. The construction depends on the following simple observation.

Lemma 1. Let $K$ be a set of real numbers, and let $a \in \mathbf{Z} \backslash\{0\}$. Then $a \in K-K$ if and only if there is a two-element subset $\{x, y\}$ of $K$ such that $(x)=(y)$ and $a=[x]-[y]$.

Proof. For any non-zero integer $a$, we have $a \in K-K$ if and only if there exist $x, y \in K$ such that $x \neq y$ and

$$
a=x-y=[x]-[y]+(x)-(y) .
$$

Since $[x]-[y] \in \mathbf{Z}$ and $-1<(x)-(y)<1$, it follows that $(x)-(y)=0$ and $a=[x]-[y]$. The set $\{x, y\}$ satisfies the conditions of the Lemma.

Here are three examples. We associate the set $A_{1}=\{2,5\}$ with the $\mathcal{N}$-set

$$
K\left(A_{1}\right)=[0,1 / 3] \cup[2+1 / 3,2+2 / 3] \cup[4+2 / 3,5] .
$$

There are three two-element subsets $\{x, y\}$ of $K\left(A_{1}\right)$ such that $x$ and $y$ have the same fractional part: $\{1 / 3,2+1 / 3\},\{2+2 / 3,4+2 / 3\}$, and $\{0,5\}$.

The set $A_{2}=\{6,10,15\}$ arises from the $\mathcal{N}$-set

$$
K\left(A_{2}\right)=[0,1 / 3] \cup[15+1 / 3,15+2 / 3] \cup[9+2 / 3,10] .
$$

The complete list of the two-element subsets $\{x, y\}$ of $K\left(A_{2}\right)$ such that $x$ and $y$ have the same fractional part is: $\{1 / 3,15+1 / 3\},\{15+2 / 3,9+2 / 3\}$, and $\{0,10\}$. 
For the set $A_{3}=\{18,28,63\}$, the $\mathcal{N}$-set

$$
\begin{gathered}
K\left(A_{3}\right)=\bigcup_{i=0}^{9}[-18 i+i / 13,-18 i+(i+1) / 13] \cup[-99+10 / 13,-99+11 / 13] \\
\cup[-36+11 / 13,-36+12 / 13] \cup[27+12 / 13,28]
\end{gathered}
$$

satisfies

$$
\left(K\left(A_{3}\right)-K\left(A_{3}\right)\right) \cap \mathbf{N}=A_{3} .
$$

There are exactly 12 two-element subsets $\{x, y\}$ of $K\left(A_{3}\right)$ such that $x$ and $y$ have the same fractional part.

In the following Lemma we construct an important example of an $\mathcal{N}$-set on the real line, and its associated difference set of integers.

Lemma 2. For the positive integer $w$, let

$$
\lambda_{0}<\lambda_{1}<\cdots<\lambda_{w-1}<\lambda_{w}
$$

be a strictly increasing sequence of real numbers such that

$$
\lambda_{w}=\lambda_{0}+1
$$

and let $b_{0}, b_{1}, \ldots, b_{w-1}$ be a sequence of integers such that

$$
b_{k-1} \neq b_{k} \text { for } k=1, \ldots, w-1
$$

and

$$
1+b_{w-1} \neq b_{0} .
$$

The set

is an $\mathcal{N}$-set, and

$$
K^{\prime}=\bigcup_{k=0}^{w-1}\left[b_{k}+\lambda_{k}, b_{k}+\lambda_{k+1}\right]
$$

$$
\left(K^{\prime}-K^{\prime}\right) \cap \mathbf{N}=\left\{\left|b_{k}-b_{k-1}\right|: k=1, \ldots, w-1\right\} \cup\left\{\left|1+b_{w-1}-b_{0}\right|\right\}
$$

is a finite set of relatively prime positive integers.

Proof. The set $K^{\prime}$ is compact because it is a finite union of closed intervals, and an $\mathcal{N}$-set because

$$
\bigcup_{k=0}^{w-1}\left[\lambda_{k}, \lambda_{k+1}\right]=\left[\lambda_{0}, \lambda_{w}\right]=\left[\lambda_{0}, \lambda_{0}+1\right] .
$$

Let $A$ be the finite set of positive integers contained in the difference set $K^{\prime}-K^{\prime}$. Since

$$
\left\{\left\{b_{k-1}+\lambda_{k}, b_{k}+\lambda_{k}\right\}: k=1, \ldots, w-1\right\} \cup\left\{\left\{b_{0}+\lambda_{0}, b_{w-1}+\lambda_{w}\right\}\right\}
$$

is the set of all two-element subsets $\{x, y\}$ of $K^{\prime}$ with $(x)=(y)$, it follows that

$$
A=\left(K^{\prime}-K^{\prime}\right) \cap \mathbf{N}=\left\{\left|b_{k}-b_{k-1}\right|: k=1, \ldots, w-1\right\} \cup\left\{\left|1+b_{w-1}-b_{0}\right|\right\} .
$$

Choose $\varepsilon_{k} \in\{1,-1\}$ such that

$$
\left|b_{k}-b_{k-1}\right|=\varepsilon_{k}\left(b_{k}-b_{k-1}\right)
$$

for $k=1, \ldots, w-1$, and $\varepsilon_{w} \in\{1,-1\}$ such that

$$
\left|1+b_{w-1}-b_{0}\right|=\varepsilon_{w}\left(1+b_{w-1}-b_{0}\right) .
$$


Since

$$
1=\varepsilon_{w}\left|1+b_{w-1}-b_{0}\right|-\sum_{k=1}^{w-1} \varepsilon_{k}\left|b_{k}-b_{k-1}\right|
$$

it follows that $A$ is a finite set of relatively prime positive integers.

Theorem 2. If $A$ is a finite set of relatively prime positive integers, then there is an $\mathcal{N}$-set $K$ such that $A=(K-K) \cap \mathbf{N}$.

Proof. Since the elements of $A$ are relatively prime, we can write 1 as an integral linear combination of elements of $A$. Thus, there exist pairwise distinct integers $a_{1}, \ldots, a_{h}$ in $A$, positive integers $w_{1}, \ldots, w_{h}$, and $\varepsilon_{1}, \ldots, \varepsilon_{h} \in\{1,-1\}$ such that

$$
\sum_{i=1}^{h} \varepsilon_{i} w_{i} a_{i}=1
$$

Rewriting (11), we obtain

$$
\varepsilon_{h} a_{h}=1+\sum_{i=1}^{h-1} w_{i}\left(-\varepsilon_{i} a_{i}\right)+\left(w_{h}-1\right)\left(-\varepsilon_{h} a_{h}\right) .
$$

Let $w_{0}=0$ and $w=\sum_{i=1}^{h} w_{i}$. For $j=1,2, \ldots, w$, we define integers $\tilde{a}_{j}$ as follows: If

$$
w_{1}+\cdots+w_{i-1}+1 \leq j \leq w_{1}+\cdots+w_{i-1}+w_{i}
$$

then

It follows that

$$
\tilde{a}_{j}=-\varepsilon_{i} a_{i} .
$$

$$
1+\sum_{j=1}^{w} \tilde{a}_{j}=1+\sum_{i=1}^{h} w_{i}\left(-\varepsilon_{i} a_{i}\right)=0 .
$$

For $k=0,1, \ldots, w$, we consider the integers

$$
b_{k}=\sum_{j=1}^{k} \tilde{a}_{j}
$$

and real numbers

$$
\lambda_{k}=\frac{k}{w}
$$

Then $b_{0}=0$,

$$
0=\lambda_{0}<\lambda_{1}<\cdots<\lambda_{w}=1
$$

and, for $k=1, \ldots, w$,

$$
b_{k}-b_{k-1}=\tilde{a}_{k} \neq 0 .
$$

It follows from (11) and (3) that

$$
\begin{aligned}
1+b_{w-1} & =1+\sum_{j=1}^{w-1} \tilde{a}_{j}=1+\sum_{j=1}^{w} \tilde{a}_{j}-\tilde{a}_{w} \\
& =-\tilde{a}_{w}=\varepsilon_{h} a_{h} \neq 0=b_{0} .
\end{aligned}
$$

Construct the $\mathcal{N}$-set

$$
K^{\prime}=\bigcup_{k=0}^{w-1}\left[b_{k}+\lambda_{k}, b_{k}+\lambda_{k+1}\right]
$$


By Lemma 2,

$$
\begin{aligned}
\left(K^{\prime}-K^{\prime}\right) \cap \mathbf{N} & =\left\{\left|b_{k}-b_{k-1}\right|: k=1, \ldots, w-1\right\} \cup\left\{\left|1+b_{w-1}-b_{0}\right|\right\} \\
& =\left\{\left|\tilde{a}_{k}\right|: k=1, \ldots, w-1\right\} \cup\left\{\left|-\tilde{a}_{w}\right|\right\} \\
& =\left\{a_{i}: i=1, \ldots, h\right\} .
\end{aligned}
$$

Let $\operatorname{card}(A)=\ell$. If $\ell=h$, then $A=\left\{a_{1}, \ldots, a_{h}\right\}$ and we set $K=K^{\prime}$.

Suppose that $\ell>h$ and $A \backslash\left\{a_{1}, \ldots, a_{h}\right\}=\left\{a_{h+1}, \ldots, a_{\ell}\right\} \neq \emptyset$. Since

$$
\frac{i}{w(\ell-h+1)} \in\left[0, \frac{1}{w}\right]=\left[b_{0}+\lambda_{0}, b_{0}+\lambda_{1}\right] \subseteq K^{\prime}
$$

for $i=1,2, \ldots, \ell-h$, it follows that

$$
K=K^{\prime} \cup\left\{a_{h+i}+\frac{i}{w(\ell-h+1)}: i=1,2, \ldots, \ell-h\right\}
$$

is an $\mathcal{N}$-set such that $A=(K-K) \cap \mathbf{N}$. This completes the proof.

Let $A$ be a finite set of relatively prime positive integers. We define the weight of a representation of 1 in the form (1) by

$$
\sum_{i=1}^{h} w_{i}+\operatorname{card}(A)-h
$$

We define the additive weight of $A$, denoted $\operatorname{Add}(A)$ as the smallest weight of a representation of 1 by elements of $A$. Note that $\operatorname{Add}(A) \geq \operatorname{card}(A)$ for all $A$, and $\operatorname{Add}(A)=\operatorname{card}(A)$ if and only if there exist distinct integers $a_{1}, \ldots, a_{h} \in A$ and $\varepsilon_{1}, \ldots, \varepsilon_{h} \in\{1,-1\}$ such that $\sum_{i=1}^{h} \varepsilon_{i} a_{i}=1$.

We define the weight of an $\mathcal{N}$-set $K$ as the number of connected components of $K$, and the geometric weight of $A$, denoted $\operatorname{Geo}(A)$ as the smallest weight of an $\mathcal{N}$-set $K$ such that $A=(K-K) \cap \mathbf{N}$.

The following result follows immediately from the proof of Theorem 2 .

Corollary 1. Let $A$ be a finite set of relatively prime positive integers. Then

$$
\operatorname{Geo}(A) \leq \operatorname{Add}(A)
$$

There exist sets $A$ such that $\operatorname{Geo}(A)<\operatorname{Add}(A)$. For example, if $A=\{1,2,3, \ldots, n\}$, then $K=[0, n]$ is an $\mathcal{N}$-set of weight 1 such that $(K-K) \cap \mathbf{N}=A$, and so $\operatorname{Geo}(A)=1<n=\operatorname{Add}(A)$.

\section{Relatively PRIME SETS OF LATTICE POINTS}

In this section we obtain the converse of Theorem 2 .

Theorem 3. If $K$ is an $\mathcal{N}$-set in $\mathbf{R}$, then $A=(K-K) \cap \mathbf{N}$ is a finite set of relatively prime positive integers.

We prove this result in $n$ dimensions.

Theorem 4. If $K$ is an $\mathcal{N}$-set in $\mathbf{R}^{n}$, then $A=(K-K) \cap \mathbf{Z}^{n}$ is a finite set of relatively prime lattice points.

Note the necessity of the compactness condition. For $n \geq 1$, the non-compact set $K=[0,1)^{n}$ has the property that for all $x \in \mathbf{R}^{n}$ there exists $y \in K$ with $x \equiv y$ $\left(\bmod \mathbf{Z}^{n}\right)$, but $(K-K) \cap \mathbf{Z}^{n}=\{0\}$. 
Proof. The proof uses a result called "the fundamental observation of geometric group theory" (Theorem 5). We discuss this in Appendix A.

The additive group $\mathbf{Z}^{n}$ acts isometrically and properly discontinuously on $\mathbf{R}^{n}$ by translation: $(g, x) \mapsto g+x$ for $g \in \mathbf{Z}^{n}$ and $x \in \mathbf{R}^{n}$. The quotient space $\mathbf{Z}^{n} \backslash \mathbf{R}^{n}$ is the $n$-dimensional torus, which is compact, and so the group action $\mathbf{Z}^{n} \curvearrowright \mathbf{R}^{n}$ is co-compact. Let $\pi: \mathbf{R}^{n} \rightarrow \mathbf{Z}^{n} \backslash \mathbf{R}^{n}$ be the quotient map. Then $\pi(x)=\langle x\rangle$ is the orbit of $x$ for all $x \in \mathbf{R}^{n}$. If $K$ is an $\mathcal{N}$-set in $\mathbf{R}^{n}$, then $K$ is compact, and for every $x \in \mathbf{R}^{n}$ there exists $y \in K$ such that $x \equiv y\left(\bmod \mathbf{Z}^{n}\right)$. This means that $\pi(y)=\langle x\rangle$, and so $\pi(K)=\mathbf{Z}^{n} \backslash \mathbf{R}^{n}$. Applying Theorem 5 to the set $K$, we conclude that the set

$$
A=\left\{a \in \mathbf{Z}^{n}: K \cap(a+K) \neq \emptyset\right\}
$$

is a finite set of generators for $\mathbf{Z}^{n}$. Moreover, $a \in A$ if and only if $a \in \mathbf{Z}^{n}$ and there exists $x \in K$ such that $x \in a+K$, that is, $x=a+y$ for some $y \in K$. Equivalently, $a \in A$ if and only if $a=x-y \in(K-K) \cap \mathbf{Z}^{n}$. This proves Theorem 4

The symmetry of the difference set immediately implies Theorem 3 .

We can state the following general inverse problem in geometric group theory: If $A$ is a finite set of generators for a group $G$ such that $A$ is symmetric and contains the identity of $G$, does there exist a geometric action of $G$ on a metric space $X$ with quotient map $\pi: X \rightarrow G \backslash X$ such that $A=\{a \in G: K \cap a K \neq \emptyset\}$ for some compact set $K$ with $\pi(K)=G \backslash X$ ? If $X$ is a group and $G$ is a subgroup of $X$ that acts on $X$ by left translation, then

$$
\{a \in G: K \cap a K \neq \emptyset\}=K K^{-1} \cap G .
$$

If $G=\mathbf{Z}^{n}$ and $X=\mathbf{R}^{n}$, then the inverse problem is to determine if every finite symmetric set relatively prime lattice points that contains 0 is of the form ( $K-$ $K) \cap \mathbf{Z}^{n}$ for some $\mathcal{N}$-set $K$. In this paper we proved that the answer is "yes" for $G=\mathbf{Z}$, but the answer is not known for higher dimension. In particular, the inverse problem for lattice points is open for $n=2$. We would like a description of the sets of lattice points that can be represented in the form $(K-K) \cap \mathbf{Z}^{2}$ for some $\mathcal{N}$-set $K$.

\section{Appendix A. The Fundamental observation of GeOMEtRiC GRoup theORY}

The proof of Theorem 4 is an application of what is often called the "fundamental observation of geometric group theory" [1, Chapter IV, pp. 87-88]. We shall describe this result, which is not well known to number theorists.

We begin by introducing the class of boundedly compact geodesic metric spaces. The Heine-Borel theorem states that, in Euclidean space $\mathbf{R}^{n}$ with the usual metric, a closed and bounded set is compact. We shall call a metric space $(X, d)$ boundedly compact if every closed and bounded subset of $X$ is compact. Equivalently, $X$ is boundedly compact if every closed ball

$$
B^{*}\left(x_{0}, r\right)=\left\{x \in X: d\left(x_{0}, x\right) \leq r\right\}
$$

is compact for all $x_{0} \in X$ and $r \geq 0$. Boundedly compact metric spaces are also called proper metric spaces.

A metric space $(X, d)$ is geodesic if, for all points $x_{0}, x_{1} \in X$ with $x_{0} \neq x_{1}$, there is an isometry $\gamma$ from an interval $[a, b] \subseteq \mathbf{R}$ into $X$ such that $\gamma(a)=x_{0}$ and $\gamma(b)=x_{1}$. Thus, if $t, t^{\prime} \in[a, b]$, then $d\left(\gamma(t), \gamma\left(t^{\prime}\right)\right)=\left|t-t^{\prime}\right|$. In particular, 
$d\left(x_{0}, x_{1}\right)=d(\gamma(a), \gamma(b))=b-a$. For example, let $x_{0}, x_{1} \in \mathbf{R}^{n}$ with $\left|x_{1}-x_{0}\right|=T$. Define $\gamma:[0, T] \rightarrow \mathbf{R}^{n}$ by

$$
\gamma(t)=x_{0}+\frac{t}{T}\left(x_{1}-x_{0}\right)
$$

Then $\gamma(0)=x_{0}, \gamma(T)=x_{1}$, and

$$
\begin{aligned}
\left|\gamma(t)-\gamma\left(t^{\prime}\right)\right| & =\left|\left(x_{0}+\frac{t}{T}\left(x_{1}-x_{0}\right)\right)-\left(x_{0}+\frac{t^{\prime}}{T}\left(x_{1}-x_{0}\right)\right)\right| \\
& =\left|\left(\frac{t-t^{\prime}}{T}\right)\left(x_{1}-x_{0}\right)\right|=\left|t-t^{\prime}\right| .
\end{aligned}
$$

Thus, $\mathbf{R}^{n}$ is a boundedly compact geodesic metric space.

Let $G$ be a group that acts on a metric space $(X, d)$. We say that the group $G$ acts isometrically on $X$ if the function $x \mapsto g x$ is an isometry on $X$ for every $g \in G$. The group action is called properly discontinuous if, for every compact subset $K$ of $X$, there are only finitely many $a \in G$ such that $K \cap a K \neq \emptyset$. Let $A=\{a \in G: K \cap a K \neq \emptyset\}$. Then $A \neq \emptyset$ because $e \in A$. Since

$$
K \cap a^{-1} K=a^{-1}(K \cap a K)
$$

it follows that $A^{-1}=A$.

For every element $x_{0} \in X$, the orbit of $x_{0}$ is the set

$$
\left\langle x_{0}\right\rangle=\left\{g x_{0}: g \in G\right\}=G x_{0} .
$$

The orbits of elements of $X$ partition the set $X$. Let $G \backslash X$ denote the set of orbits of the group action, and define the function $\pi: X \rightarrow G \backslash X$ by $\pi(x)=\langle x\rangle$. We call $G \backslash X$ the quotient space of $X$ by $G$, and we call $\pi$ the quotient map of $X$ onto $G \backslash X$. Note that every orbit $\langle x\rangle$ is a subset of the set $X$ and a point in the quotient space $G \backslash X$.

We define the quotient topology on $G \backslash X$ as follows: A set $V$ in $G \backslash X$ is open if and only if $\pi^{-1}(V)$ is open in $X$. This is the largest topology on the quotient space $G \backslash X$ such that the quotient map $\pi$ is continuous. We call the group action $G \curvearrowright X$ co-compact if the quotient space $G \backslash X$ is compact. An isometric, properly discontinuous, co-compact action of a group $G$ on a boundedly compact geodesic metric space is called a geometric action.

We now state the "fundamental observation of geometric group theory."

Theorem 5. Let $(X, d)$ be a boundedly compact geodesic metric space and let $G$ be a group that acts isometrically on $X$. Suppose that the group action $G \curvearrowright X$ is properly discontinuous and co-compact. Let $\pi: X \rightarrow G \backslash X$ be the quotient map, and let $K$ be a compact subset of $X$ such that $\pi(K)=G \backslash X$. Then

$$
A=\{a \in G: K \cap a K \neq \emptyset\}
$$

is a finite set of generators for $G$.

For example, the additive group $\mathbf{Z}^{n}$ of $n$-dimensional lattice points acts on Euclidean space $\mathbf{R}^{n}$ by translation: $\alpha_{g}(x)=g+x$ for $g \in \mathbf{Z}^{n}$ and $x \in \mathbf{Z}^{n}$. The group $\mathbf{Z}^{n}$ acts isometrically on $\mathbf{R}^{n}$ since

$$
\left|\alpha_{g}(x)-\alpha_{g}(y)\right|=|(g+x)-(g+y)|=|x-y| .
$$


Let $K$ be a compact subset of $\mathbf{R}^{n}$. Then $K$ is bounded and there is a number $r>0$ such that $|x|<r$ for all $x \in K$. If $g \in \mathbf{Z}^{n}$ and $K \cap(g+K) \neq \emptyset$, then there exists $x \in K$ such that $g+x \in K$. Therefore,

$$
|g|-r<|g|-|x| \leq|g+x|<r
$$

and $|g|<2 r$. There exist only finitely many lattice points in $\mathbf{Z}^{n}$ of length less than $2 r$, and so the action on $\mathbf{Z}^{n}$ on $\mathbf{R}^{n}$ is properly discontinuous.

We shall prove that the group action $\mathbf{Z}^{n} \curvearrowright \mathbf{R}^{n}$ is co-compact. Let $\pi: \mathbf{R}^{n} \rightarrow$ $\mathbf{Z}^{n} \backslash \mathbf{R}^{n}$ be the quotient map. The quotient space $\mathbf{T}^{n}=\mathbf{Z}^{n} \backslash \mathbf{R}^{n}$ is called the $n$ dimensional torus. Let $\left\{W_{i}\right\}_{i \in I}$ be an open cover of $\mathbf{T}^{n}$, and define $V_{i}=\pi^{-1}\left(W_{i}\right)$ for all $i \in I$. Then $\left\{V_{i}\right\}_{i \in I}$ is an open cover of $\mathbf{R}^{n}$. The unit cube

$$
K=[0,1]^{n}=\left\{x=\left(x_{1}, \ldots, x_{n}\right) \in \mathbf{R}^{n}: 0 \leq x_{i} \leq 1 \text { for all } i=1, \ldots, n\right\}
$$

is a compact subset of $\mathbf{R}^{n}$, and $\pi(K)=\mathbf{T}^{n}$. Since $\left\{V_{i}\right\}_{i \in I}$ is an open cover of $K$, it follows that there is a finite subset $J$ of $I$ such that $K \subseteq \bigcup_{j \in J} V_{j}$, and so

$$
\mathbf{T}^{n}=\pi(K) \subseteq \bigcup_{j \in J} \pi\left(V_{j}\right)=\bigcup_{j \in J} W_{j} .
$$

Therefore, $\mathbf{T}^{n}$ is compact and the group action $\mathbf{Z}^{n} \curvearrowright \mathbf{R}^{n}$ is co-compact.

\section{REFERENCES}

[1] P. de la Harpe, Topics in Geometric Group Theory, Chicago Lectures in Mathematics, University of Chicago Press, Chicago, IL, 2000.

[2] V. A. Efremovič, The proximity geometry of Riemannian manifolds, Uspekhi Mat. Nauk 8 (1953), 189.

[3] J. Milnor, A note on curvature and fundamental group, J. Differential Geometry 2 (1968), $1-7$.

[4] M. B. Nathanson, Phase transitions in infinitely generated groups, and related problems in additive number theory, arXiv: 0811.3990, 2008.

[5] - Nets in groups, minimum length g-adic representations, and minimal additive complements, arXiv: 0812.0560, 2008.

[6] _ Bi-Lipschitz equivalent metrics on groups, and a problem in additive number theory, arXiv: 0902.3254, 2009.

[7] A. S. Švarc, A volume invariant of coverings, Dokl. Akad. Nauk SSSR (N.S.) 105 (1955), $32-34$.

Department of Mathematics, Lehman College (CUNY), Bronx, New York 10468

E-mail address: melvyn.nathanson@lehman.cuny.edu 